Received: 2020/11/22, Revised: 2020/12/24, Accepted: 2020/12/30, Published: 2021/03/31 @2021 Aoi Mamiya et al.; Licence Physical Activity and Nutrition. This is an open access article distributed under the terms of the creative commons attribution license (https:/l creativecommons.org/licenses/by-nc/2.0/), which permits unrestricted use, distribution, and reproduction in any medium, provided the orginal work is properly cited.

${ }^{*}$ Corresponding author : Kazushige Goto, Ph.D.

Professor, Graduate School of Sports and Health Science Ritsumeikan University, 1-1-1, Nojihigashi, Kusatsu, Shiga 525-8577, Japan.

Tel: +81-77-599-4127 / Fax: +81-77-599-4127

E-mail: kagoto@fc.ritsumei.ac.jp

@2021 The Korean Society for Exercise Nutrition

\section{Effects of partial sleep deprivation after prolonged exercise on metabolic responses and exercise performance on the following day}

\author{
Aoi Mamiya / Ikuhiro Morii / Kazushige Goto* \\ Graduate School of Sports and Health Science, Ritsumeikan University, Shiga, Japan
}

\section{INTRODUCTION}

Athletes are required to perform strenuous exercise on consecutive days to improve their performance. Therefore, it is essential for them to promote recovery of physical function (e.g., maximal strength and power output) during the post-exercise period to increase the quality of their next training session. Among the various "post-exercise treatments" that have been established, including massage, cryotherapy, nutrient intake, and sleep ${ }^{1-3}$, sleep stands out as it is indispensable for life, physiological growth, and repair ${ }^{3}$. In general, 9-10 h of sleep per night is recommended to facilitate appropriate recovery following an exercise session ${ }^{4}$. However, a survey of 890 elite athletes revealed that $41 \%$ of the athletes experienced sleep problems (e.g., falling asleep at night) and 11\% slept less than $6 \mathrm{~h}$ per night ${ }^{5}$. Furthermore, the onset of sleep among athletes can be delayed due to stress derived either from competition, daily strenuous exercise, or training and travel occurring late in the evening ${ }^{6-8}$. According to Oda and Shirakawa ${ }^{8}$, high-intensity exercise before bedtime delayed sleep onset relative to moderate-intensity exercise.

Total sleep deprivation (TSD) and partial sleep deprivation (PSD) have been shown to impair exercise performance ${ }^{9-13}$. Oliver et al. ${ }^{12}$ showed that a single night of sleep deprivation decreased performance during an endurance running test on the following day. Moreover, maximal aerobic power decreased by $50 \%$ after PSD $^{11}$, while heart rate and minute ventilation during submaximal exercise increased ${ }^{10}$. Peak oxygen consumption during exercise also decreased significantly, although the maximal workload at exhaustion was not affected. In addition to impairing endurance performance, PSD may also reduce anaerobic performance. It was found that $4 \mathrm{~h}$ of PSD significantly reduced both peak and mean power outputs during a $30 \mathrm{~s}$ maximal sprint ${ }^{9}$. Furthermore, PSD augmented exercise-induced elevations in plasma interleukin-6 (IL6 ) and tumor necrosis factor-alpha (TNF- $\alpha$ ) following repeated sprint training ${ }^{14}$, and a single night of PSD increased the transcription of IL-6 and TNF- $\alpha^{15}$. However, these experiments were designed to determine the impact of reduced sleep duration itself, without exercise. Considering 
that athletes perform consecutive days of high-intensity exercise, it is necessary to determine the impact of PSD after an exercise session. Therefore, the present study examined the effect of PSD after exercise on metabolic responses and exercise performance on the following morning. We hypothesized that a single night of PSD after exercise would reduce exercise performance when compared with a normal sleep duration.

\section{METHODS}

\section{Subjects}

Eleven male athletes participated in the study. The mean \pm standard error (SE) age, height, and body mass of the study subjects were $20.8 \pm 0.8$ years, $168.9 \pm 6.9 \mathrm{~cm}, 67.4 \pm 9.8$ $\mathrm{kg}$, respectively. They all followed a regular sleep cycle, with an average sleep duration of approximately $6 \mathrm{~h}$ and 7 min. Prior to the study, all subjects were informed about the experimental procedures and possible risks involved in this study, and they subsequently provided informed consent. The present study was approved by the Ethics Committee for Human Experiments at Ritsumeikan University, Shiga, Japan.

\section{Experimental design}

Habitual sleep duration was monitored for 7 days before the experiment. Sleep duration was recorded using an accelerometer (ActiGraph; Ambulatory Monitoring, Inc., Ardsley, NY, USA), and the individual sleep duration and magnitude of sleep deprivation were determined. Prior to the experiment, an incremental running test was performed to evaluate the maximal oxygen uptake $\left(\dot{\mathrm{V}}_{2 \max }\right)$ using a treadmill (Valient; Lode, Groningen, the Netherlands). On day 1 of the experiment, the subjects arrived at the laboratory at 16:00 and performed an exercise session. They then completed one of the two trials in which they were allowed different amounts of sleep. For the control (CON) trial, the subjects slept from 23:00 to 7:00, whereas for the PSD trial, the subjects' sleep duration was shortened to $40 \%$ of their individual sleep duration (a $60 \%$ reduction of the normal sleep duration). In the PSD trial, the early phase of nighttime sleep was deprived. During this period of sleep deprivation, the subjects were not allowed to sleep, and they spent this time watching television, reading books, or engaging in other non-exercise activities. On the following morning (day 2) of both trials, exercise performance and subjective scores of fatigue, leg muscle soreness, sleepiness, quality of sleep, and falling asleep were evaluated (Figure 1). The two trials were separated by a 3-week interval using a randomized crossover design. The subjects' diet and physical activity levels were controlled to match in the two trials.

\section{Exercise protocol}

On day 1, the exercise protocol was started with a $5 \mathrm{~min}$ warm-up consisting of running at $60 \% \dot{\mathrm{VO}}_{2 \max }$. Then, the subjects completed $90 \mathrm{~min}$ of continuous running at $75 \%$ $\mathrm{VO}_{2 \max }$ followed by drop jumps $(10 \times 10$ sets, 100 drop jumps in total). They were allowed a 5 min rest between the 90 min of continuous running and the drop jumps. The maximal voluntary isometric contraction (MVC) of knee extension was evaluated before and after completion of the drop jumps. On the following morning (day 2), the MVC was evaluated again, followed by $20 \mathrm{~min}$ of submaximal running at $75 \% \mathrm{VO}_{2 \max }$ and a time to exhaustion (TTE) test at $85 \% \mathrm{VO}_{2 \max }$.

\section{Measurements}

$\dot{\mathrm{V}} \mathrm{O}_{2 \max }$

The running velocity was initially set at $6 \mathrm{~km} / \mathrm{h}$, and then was increased by $2 \mathrm{~km} / \mathrm{h}$ every $4 \mathrm{~min}$ until it reached 10 $\mathrm{km} / \mathrm{h}$. Once the running velocity reached $10 \mathrm{~km} / \mathrm{h}$, it was increased by $2 \mathrm{~km} / \mathrm{h}$ every $3 \mathrm{~min}$ until it reached $14 \mathrm{~km} / \mathrm{h}$. Once the running velocity reached $14 \mathrm{~km} / \mathrm{h}$, it was increased by $0.6 \mathrm{~km} / \mathrm{h}$ every minute until volitional exhaustion. During the test, expired gases were collected and analyzed with an automatic gas analyzer (AE300S; Minato Medical Science Co., Ltd., Tokyo, Japan). The collected data were

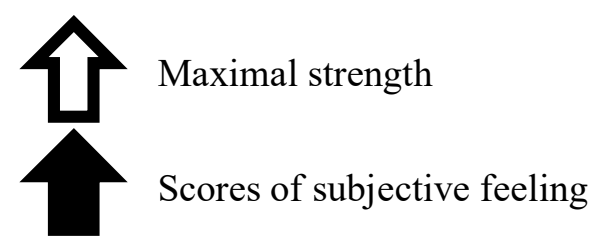

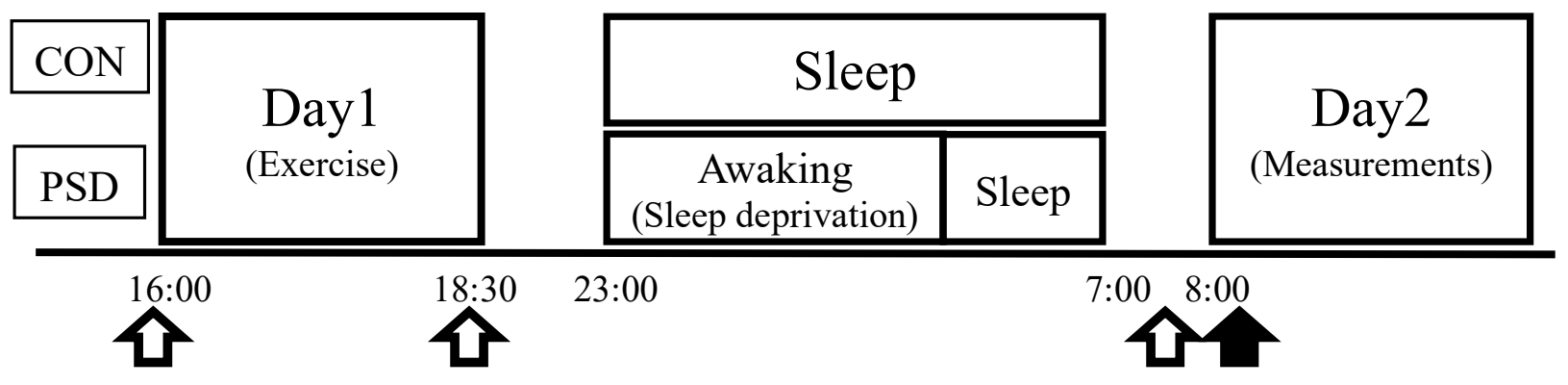

Figure 1. Timeline of the main experiment on days 1 and 2. 
averaged every $30 \mathrm{~s}$. Heart rate (HR) was measured continuously during the test with a wireless HR monitor (Acculex Plus; Polar Electro Oy, Kempele, Finland).

\section{Maximal voluntary isometric contraction of knee extension}

The MVC with the knee positioned at $70^{\circ}$ of extension (the fully extended position was defined as $0^{\circ}$ ) was measured using an isokinetic dynamometer (Biodex System 4; SAKAI Medical Co., Tokyo, Japan). The subjects exerted their maximal strength (3s) knee extension twice, and the highest value was included in the analysis. A $60 \mathrm{~s}$ rest period was provided between extensions.

\section{Subjective assessments}

Subjective fatigue, leg muscle soreness, sleepiness, quality of sleep, and falling asleep were evaluated using a 100 $\mathrm{mm}$ visual analog scale. The subjects provided ratings for respiratory strain (RPE-R) and leg strain (RPE-L) every 15 min during the running sessions on days 1 and 2 using a 10 -point scale of perceived exertion ${ }^{16}$.

\section{Physiological responses}

On day 2, oxygen uptake $\left(\dot{\mathrm{VO}}_{2}\right)$, carbon dioxide output $\left.(\dot{\mathrm{V} C O})_{2}\right)$, respiratory exchange ratio (RER), and expired minute ventilation (VE) were determined during $20 \mathrm{~min}$ of submaximal running at $75 \% \mathrm{VO}_{2 \max }$. Respiratory samples were collected and analyzed by the breath-by-breath method using a metabolic cart (AE300S; Minato Medical Science Co., Ltd.). The collected data were averaged every $30 \mathrm{~s}$. HR was recorded at $1 \mathrm{~min}$ intervals.

\section{Time to exhaustion}

An all-out running test at $85 \% \dot{\mathrm{VO}}_{2 \max }$ to determine TTE was performed after 20 min of submaximal running at 75\% $\mathrm{VO}_{2 \max }$ and a 2 min rest. After this rest, the subjects started running again at $85 \% \mathrm{VO}_{2 \max }$ until exhaustion. No information about the elapsed time was provided to the subject during the test. The TTE was defined as the time when the subjects touched the rope twice at the prescribed position on the treadmill.

\section{Statistical analysis}

All data are expressed as the mean \pm SE. Two-way analysis of variance (ANOVA) with repeated measures was used to confirm the interaction (trial $\times$ time) and main effects (trial and time). When the ANOVA revealed a significant interaction or main effect, the Tukey-Kramer test was performed as a post-hoc analysis to identify differences. For all tests, a $p$-value less than 0.05 was considered statistically significant.

\section{RESULTS}

\section{Subjective variables}

Subjective scores of fatigue, leg muscle soreness, sleepiness, and sleep quality were evaluated on day 2 . The sleep- iness score was significantly higher in the PSD trial than in the CON trial $(p<0.001)$, whereas the vitality score was significantly lower in the PSD trial than in the CON trial ( $p$ $<0.05$ ). The scores of subjective fatigue, muscle soreness, and sleep were not significantly different between the two trials.

Cardiorespiratory variables and time to exhaustion during the submaximal running test

Figure 2 shows the change in respiratory variables during the 20 min of submaximal running on day 2 . The results show that $\dot{\mathrm{VO}}_{2}$ did not differ significantly between the $\mathrm{CON}$ and PSD trials (Figure 2A). However, as shown in Figure $2 \mathrm{~B}$ and $\mathrm{C}$, the PSD trial showed significantly lower $\mathrm{VCO}_{2}$ and RER during running $(p<0.05)$. The average RER during 20 min of running was significantly lower in the PSD trial $(0.86 \pm 0.01)$ than in the CON trial $(0.89 \pm 0.01, p$ $<0.05)$. In addition, no significant interaction between trial and time or the main effect of trial was observed for VE. The HR during running did not differ significantly at any point between the two trials.

The TTE during running at $85 \% \dot{\mathrm{VO}}_{2 \max }$ was significantly shorter in the PSD trial $(665.73 \pm 100.3 \mathrm{~s})$ than in the CON trial $(887.64 \pm 162.2 \mathrm{~s}, p<0.05$, Figure 3$)$. During the TTE test, the HR did not significantly differ between the CON $(180 \pm 5 \mathrm{bpm})$ and PSD trials $(177 \pm 4 \mathrm{bpm}, p>0.05)$.

\section{Maximal voluntary isometric contraction (MVC) of knee extension}

The changes in the MVC of knee extension are shown in Table 1. In the PSD trial, a significant decrease in MVC values was observed on day 2 when compared to day 1 (main effect for time, $p<0.05$ ), whereas no significant change was observed in the CON trial. However, no significant difference between the PSD and CON trials was observed at any time point.

Table 1. MVC of knee extension on Day 1 and Day 2.

\begin{tabular}{cccc} 
& $\begin{array}{c}\text { Day 1 } \\
\text { (Before exercise) }\end{array}$ & $\begin{array}{c}\text { Day 1 } \\
\text { (After exercise) }\end{array}$ & Day 2 \\
\hline CON & $225 \pm 8$ & $188 \pm 10^{*}$ & $206 \pm 9$ \\
\hline PSD & $224 \pm 11$ & $199 \pm 9$ & $187 \pm 9^{*}$ \\
\hline
\end{tabular}

Values are means \pm SE. Unit: Nm. ${ }^{*} ; P<0.05$ vs. before exercise on Day 1.

\section{DISCUSSION}

The present study investigated the effect of PSD after a prolonged exercise session on exercise performance on the following morning. The results showed that neither the $\dot{\mathrm{VO}}_{2}$ (running economy) during 20 min of running at $75 \% \mathrm{VO}_{2 \max }$ nor the MVC differed significantly between the PSD and CON trials. A novel finding of this study is that the RER during running (at $75 \% \dot{\mathrm{VO}}_{2 \max }$ ) was significantly lower in the PSD trial than in the CON trial. In addition, the TTE during subsequent running at $85 \% \dot{\mathrm{VO}}_{2 \max }$ was significantly shortened in the PSD trial when compared to the CON trial. 


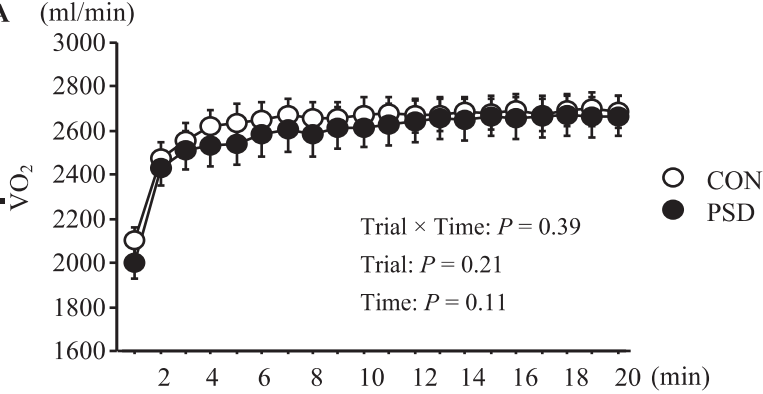

B

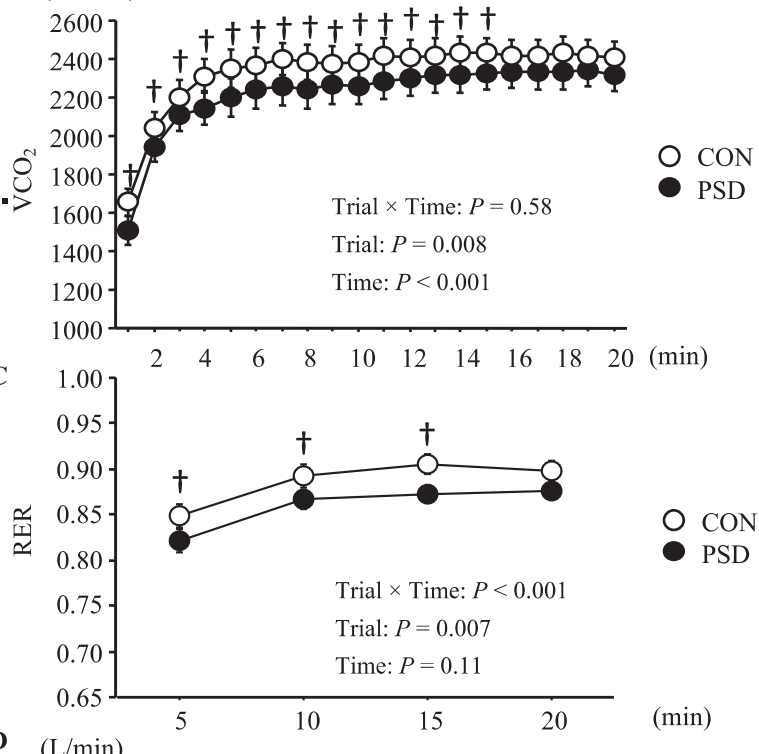

D $(\mathrm{L} / \mathrm{min})$

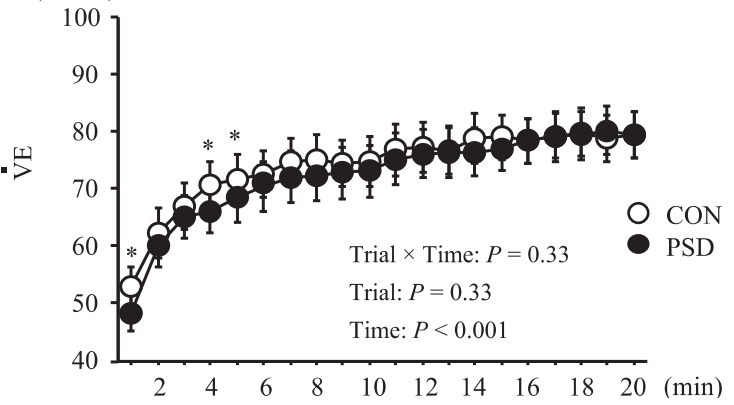

Figure 2. Oxygen uptake $\left(\dot{\mathrm{V}}_{2}\right)(\mathrm{A})$, carbon dioxide output $\left(\dot{\mathrm{V}} \mathrm{CO}_{2}\right)(\mathrm{B})$ respiratory exchange ratio (RER) (C), and expired minute ventilation (VE) (D) during 20 min of running at $75 \% \mathrm{VO}_{2 \max }$ in the control (CON) and partia sleep deprivation (PSD) trials. Values are means $\pm \mathrm{SE}$. $t ; P<0.05$ between rials.

These findings indicate that PSD after an exercise session reduces endurance performance, but not maximal muscular strength, on the following morning.

The RER during 20 min of submaximal running at $75 \%$ $\mathrm{VO}_{2 \max }$ was significantly lower in the PSD trial, suggesting augmented fat utilization during the exercise. In general, augmented fat utilization during submaximal exercise decreases muscle glycogen utilization (i.e., muscle glycogen is spared due to augmented fat utilization), leading to an increase in TTE during exercise ${ }^{17}$. In contrast, the PSD trial revealed a significantly shorter TTE at $85 \% \dot{\mathrm{VO}}_{2 \max }$ after 20 min of submaximal running at $75 \% \dot{\mathrm{VO}}_{2 \max }$. A plausible reason for the impaired endurance performance in the PSD (s)

$\dagger$

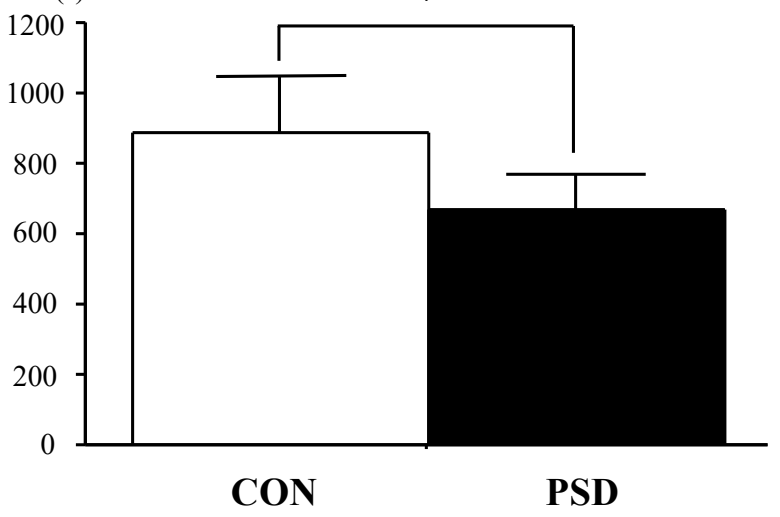

Figure 3. Time to exhaustion (TTE) during running at $85 \% \dot{V}_{2 \max }$ in the control (CON) and partial sleep deprivation (PSD) trials. Values are mean \pm SE. $\dagger, P<0.05$ vs. CON.

trial is reduced muscle glycogen content at the start of the exercise on day 2. Skein et al. ${ }^{18}$ investigated the influence of a single night of sleep deprivation on muscle glycogen content and intermittent sprint performance on the following morning. In that study, team sport athletes conducted a 30 min incremental run followed by intermittent sprint exercises after $30 \mathrm{~h}$ of sleep deprivation. Muscle biopsy samples were collected before exercise. Sleep deprivation decreased intermittent sprint performance along with a concomitant reduction in muscle glycogen content before exercise. Moreover, in a another study, performance of a 3 $\mathrm{km}$ time trial (3 km TT) was impaired ( $-4 \%$ vs. the baseline on the previous day) following PSD (sleep was restricted from 22:30 to 2:00) compared with control sleep conditions $(-0.5 \% \text { vs. baseline })^{19}$. The authors suggested that the impaired $3 \mathrm{~km}$ TT performance was attributed to incomplete muscle glycogen replenishment following exercise in the previous evening. Sleep deprivation may also affect glucose metabolism during exercise. As mentioned previously, the RER during 20 min of submaximal exercise was significantly lower in the PSD trial. Exercise with decreased glycogen content impairs exercise tolerance ${ }^{20}$, but a compensatory increase in fat utilization (shown as a lower RER) would be induced. Recovery of muscle glycogen content during the post-exercise period is strongly influenced by energy intake after the completion of exercise ${ }^{21}$. In the present study, diet during post-exercise recovery was carefully controlled and was kept constant between the two trials. Therefore, it is unlikely that the difference in energy intake affected the results. However, because energy expenditure during sleep is lower than that during waking ${ }^{22}$, energy expenditure during the night was considered to be higher in the PSD trial than in the CON trial. It is also interesting that the VE during the initial part of the 20 min run was significantly lower in the PSD trial than in the CON trial. Therefore, PSD appeared to attenuate the initial increase in the cardiorespiratory response during exercise ${ }^{23}$. In contrast, no change in HR was observed during $20 \mathrm{~min}$ of submaximal running. This is consistent with previous findings of no change in HR during exercise following sleep deprivation ${ }^{12,24-26}$. 
Despite a marked reduction in endurance performance in the PSD trial, the influence of PSD on MVC was small, and there was no significant difference in MVC between the CON and PSD trials. In a previous study, sleep restriction after strenuous exercise did not attenuate peak isokinetic torque on the following morning ${ }^{19}$. Bambaeichi et al. ${ }^{27}$ demonstrated that PSD ( $2.5 \mathrm{~h}$ of sleep) did not significantly alter maximal strength or core temperature in the morning (6:00) or evening (18:00). Moreover, $60 \mathrm{~h}$ of sleep deprivation did not attenuate MVC compared to that under normal sleep conditions ${ }^{28}$. These findings are consistent with the results of our PSD trial.

Some limitations must be carefully considered when interpreting these results. First, we were not able to evaluate muscle glycogen content, which might explain the lower endurance performance in the PSD trial. Second, we measured the effect of PSD after an exercise session only on the following morning, thus it is unclear how long the impairment of endurance performance lasts. Third, although we focused on the impact of reduced sleep duration, sleep quality is also important for promoting recovery following exercise. Therefore, it would be meaningful to evaluate sleep quality after exercise in future studies. Finally, the present PSD trial deprived the subjects of sleep during the first part of the night. However, in some previous studies, sleep was deprived during the latter part of the night ${ }^{9,19,26}$. Studies to determine the impact of sleep deprivation in different phases of the night would be interesting.

In conclusion, a single night of PSD after exercise did not markedly affect the strength or $\dot{\mathrm{VO}}_{2}$ kinetics during submaximal running on the following morning. However, the TTE during running was significantly decreased after PSD.

\section{ACKNOWLEDGEMENTS}

We would like to thank all the subjects who participated in the study. No specific funding was received in support of this study.

\section{REFERENCES}

1. Beelen M, Burke LM, Gibala MJ, van Loon LJC. Nutritional strategies to promote postexercise recovery. Int J Sport Nutr Exerc Metab. 2010;20:515-32.

2. Krüger M, de Mareés M, Dittmar KH, Sperlich B, Mester J. Wholebody cryotherapy's enhancement of acute recovery of running performance in well-trained athletes. Int J Sports Physiol Perform. 2015;10:605-12.

3. Venter RE. Perceptions of team athletes on the importance of recovery modalities. Eur J Sport Sci. 2014;14 Suppl 1:S69-76.

4. Bompa TO, Haff GG. Periodization: theory and methodology of training. Champaign, IL: Human Kinetics, 2009.

5. Venter RE, Potgieter JR, Barnard JG. The use of recovery modalities by elite South African team athletes. South African J Res Sport, Phy Edu Recreation. 2010;34:167-84.

6. Eagles A, Mclellan C, Hing W, Carloss N, Lovell D. Changes in sleep quantity and efficiency in professional rugby union players during home-based training and match-play. J Sports Med Phys Fitness. 2016;56:565-71.

7. Fullagar HHK, Skorski S, Duffield R, Julian R, Bartlett J, Meyer T. Impaired sleep and recovery after night matches in elite football players. J Sports Sci. 2016;34:1333-9.

8. Oda S, Shirakawa K. Sleep onset is disrupted following pre-sleep exercise that causes large physiological excitement at bedtime. Eur J Appl Physiol. 2014;114:1789-99.

9. Abedelmalek S, Chtourou H, Aloui A, Aouichaoui C, Souissi N, Tabka Z. Effect of time of day and partial sleep deprivation on plasma concentrations of IL- 6 during a short-term maximal performance. Eur J Appl Physiol. 2013;113:241-8.

10. Mougin F, Simon-Rigaud ML, Davenne D, Renaud A, Garnier A, Kantelip JP, Magnin P. Effects of sleep disturbances on subsequent physical performance. Eur J Appl Physiol Occup Physiol. 1991;63:77-82.

11. Mougin F, Bourdin H, Simon-Rigaud ML, Nguyen NU, Kantelip JP, Davenne D. Hormonal responses to exercise after partial sleep deprivation and after a hypnotic drug-induced sleep. J Sports Sci. 2001;19:89-97.

12. Oliver SJ, Costa RJS, Laing SJ, Bilzon JLJ, Walsh NP. One night of sleep deprivation decreases treadmill endurance performance. Eur J Appl Physiol. 2009;107:155-61.

13. Souissi N, Sesboüé B, Gauthier A, Larue J, Davenne D. Effects of one night's sleep deprivation on anaerobic performance the following day. Eur J Appl Physiol. 2003;89:359-66.

14. Abedelmalek S, Souissi N, Chtourou H, Denguezli M, Aouichaoui C, Ajina M, Aloui A, Dogui M, Haddouk S, Tabka Z. Effects of partial sleep deprivation on proinflammatory cytokines, growth hormone, and steroid hormone concentrations during repeated brief sprint interval exercise. Chronobiol Int. 2013;30:502-9.

15. Irwin MR, Wang M, Campomayor CO, Collado-Hidalgo A, Cole S. Sleep deprivation and activation of morning levels of cellular and genomic markers of inflammation. Arch Intern Med. 2006;166:1756-62.

16. Wilson RC, Jones PW. Long-term reproducibility of borg scale estimates of breathlessness during exercise. Clin Sci. 1991;80:30912.

17. Gonzalez JT, Fuchs CJ, Betts JA, van Loon LJC. Liver glycogen metabolism during and after prolonged endurance-type exercise. Am J Physiol Endocrinol Metab. 2016;311:E543-53.

18. Skein M, Duffield R, Edge J, Short MJ, Mundel T. Intermittent-sprint performance and muscle glycogen after $30 \mathrm{~h}$ of sleep deprivation. Med Sci Sports Exerc. 2011;43:1301-11.

19. Chase JD, Roberson PA, Saunders MJ, Hargens TA, Womack CJ, Luden ND. One night of sleep restriction following heavy exercise impairs 3-km cycling time-trial performance in the morning. Appl Physiol Nutr Metab. 2017;42:909-15.

20. Balsom PD, Gaitanos GC, Söderlund K, Ekblom B. High-intensity exercise and muscle glycogen availability in humans. Acta Physiol Scand. 1999;165:337-45.

21. Murray R, Paul GL, Seifert JG, Eddy DE. Responses to varying rates of carbohydrate ingestion during exercise. Med Sci Sports Exerc. 1991;23:713-8.

22. White DP, Weil JV, Zwillich CW. Metabolic rate and breathing during sleep. J Appl Physiol. 1985;59:384-91.

23. Plyley MJ, Shephard RJ, Davis GM, Goode RC. Sleep deprivation 
and cardiorespiratory function. Influence of intermittent submaximal exercise. Eur J Appl Physiol Occup Physiol. 1987;56:338-44.

24. Azboy O, Kaygisiz Z. Effects of sleep deprivation on cardiorespiratory functions of the runners and volleyball players during rest and exercise. Acta Physiol Hung. 2009;96:29-36.

25. Martin BJ. Effect of sleep deprivation on tolerance of prolonged exercise. Eur J Appl Physiol Occup Physiol. 1981;47:345-54.

26. Mejri MA, Yousfi N, Mhenni T, Tayech A, Hammouda O, Driss T. Chaouachi A, Souissi N. Does one night of partial sleep deprivation affect the evening performance during intermittent exercise in Taekwondo players? J Exerc Rehabil. 2016;12:47-53.

27. Bambaeichi E, Reilly T, Cable NT, Giacomoni M. Influence of time of day and partial sleep loss on muscle strength in eumenorrheic females. Ergonomics. 2005;48:1499-511.

28. Symons JD, VanHelder T, Myles WS. Physical performance and physiological responses following 60 hours of sleep deprivation. Med Sci Sports Exerc. 1988;20:374-80. 